\title{
About measuring systems and measurement procedures
}

\author{
Xavier Fuentes-Arderiu
}

Received: 11 February 2011/Published online: 24 March 2011

(C) Springer-Verlag 2011

To the Editor,

The International Vocabulary of Metrology (hereafter VIM), in its last edition [1], makes an important change in the definition of the concept of 'measurement procedure.' The first and second editions of VIM [2,3] considered that a measurement procedure is a set of operations, whereas the third edition considers that a measurement procedure is a description (and a description can be understood as a document).

Contrarily, the concept of measuring system remains almost invariable in the three editions of VIM, being considered essentially as a set of measuring instruments and other equipment.

On the other hand, the current VIM [1], in Note 1 of its entry 2.24, 'reproducibility condition of measurement,' states that "different measuring systems may use different measurement procedures."

Taking into account all the above, we can conclude that for performing a measurement it is necessary to use both a measuring system and a measurement procedure. For this reason, any 'measuring system' whose performance should be evaluated (either for its validation or another sort of verification) is not defined by the set of measuring instruments alone but indispensibly requires an associated measurement procedure. To avoid any confusion, this clarification should be made explicit in any methodological description. Thus, precision, trueness, detection limit, and measuring range, among others, are metrological characteristics of a measuring system used with a specified measurement procedure.

X. Fuentes-Arderiu $(\bowtie)$

Laboratori Clínic, Hospital Universitari de Bellvitge,

08907 L'Hospitalet de Llobregat, Catalonia, Spain

e-mail: xfa@bellvitgehospital.cat
According to the same rationale, the concepts 'reference measurement procedure' (entry 2.7 in [1]) and 'primary reference measurement procedure' (entry 2.8 in [1]) should be replaced with 'reference measuring system' and 'primary reference measuring system,' respectively. And, consequently, the definition of concept 2.7 in [1] should be (proposed changes underlined) "measuring system, used with a specified measurement procedure, accepted as providing measurement results fit for their intended use in assessing measurement trueness of measured quantity values obtained from other measuring systems for quantities of the same kind, in calibration, or in characterizing reference materials," and the definition of concept 2.8 in [1] should be (proposed changes underlined) "reference measuring system, used with a specified measurement procedure, utilized to obtain a measurement result without relation to a measurement standard for a quantity of the same kind."

Independently of the performance evaluation mentioned before, as any measurement procedure usually is a written description we can only evaluate the readability of the document and validate it (or not), however obviously this is not an evaluation of a metrological characteristic.

\section{References}

1. BIPM, IEC, IFCC, ISO, IUPAC, IUPAP, OIML (2007) International vocabulary of metrology-Basic and general concepts and associated terms (VIM), 3rd Ed. ISO, Geneva [also known as ISO/ IEC Guide 99:2007, and JCGM 200:2008; http://www.bipm. org/vim]

2. BIPM IEC, ISO OIML (1984) International vocabulary of basic and general terms in metrology (VIM), 1st edn. ISO, Geneva

3. BIPM, IEC, IFCC, ILAC, ISO, IUPAC, IUPAP, OIML (1993) International vocabulary of basic and general terms in metrology (VIM), 2nd edn. ISO, Geneva 